This item was submitted to Loughborough's Institutional Repository (https://dspace.lboro.ac.uk/) by the author and is made available under the following Creative Commons Licence conditions.

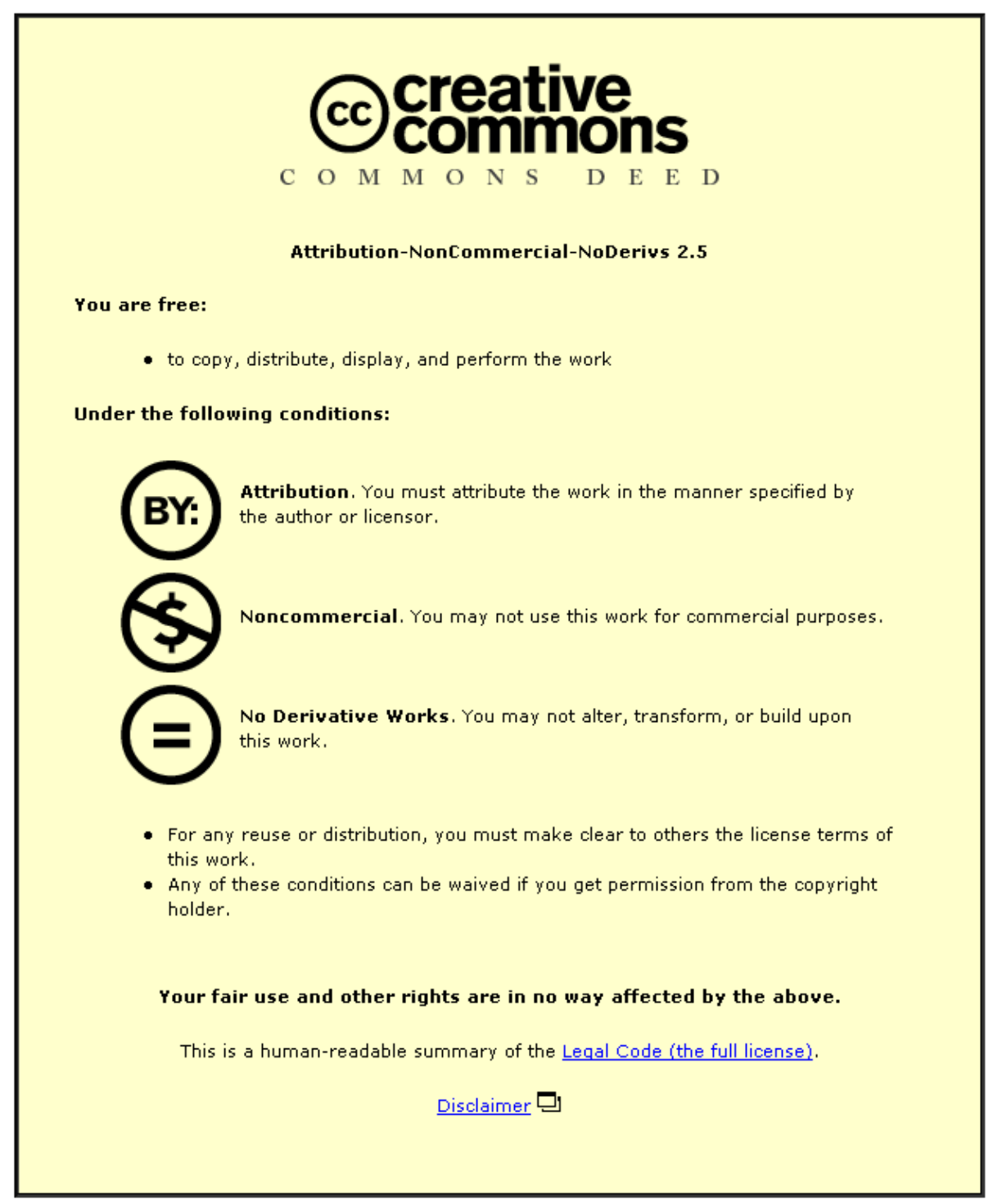

For the full text of this licence, please go to: http://creativecommons.org/licenses/by-nc-nd/2.5/ 


\title{
A GMD-Based Precoding Scheme for Downlink Multiuser Multistream MIMO Channels
}

\author{
Zhilan Xiong, Ranaji Krishna, Sangarapillai Lambotharan and Jonathon A. Chambers \\ Advanced Signal Processing Group \\ Loughborough University, Leicestershire, UK, LE11 3TU \\ Email:\{Z.Xiong, R.Krishna, S.Lambotharan, J.A.Chambers\}@lboro.ac.uk
}

\begin{abstract}
In order to obtain a good balance of bit error rate (BER) across channels, the geometric mean decomposition (GMD) is introduced to replace the singular value decomposition (SVD) for precoding in the downlink of a multiuser multistream multiple-input multiple-output (MIMO) system. By combining GMD with a block diagonalization method, we obtain two kinds of precoding schemes: iterative nullspace-directed GMD and noniterative nullspace-directed GMD. Considering their respective advantages and disadvantages, a mixed nullspace-directed GMD is proposed to solve the convergence related problems of the iterative method. Furthermore, the computational complexity of the mixed scheme is similar to the iterative scheme under the same conditions. The simulation results show that the average BER performance of the block diagonalization method based on GMD is better than the same method based on SVD, and the mixed nullspace-directed GMD outperforms the iterative nullspace-directed GMD and the non-iterative nullspace-directed GMD.
\end{abstract}

Index Terms-Geometric mean decomposition (GMD), singular value decomposition (SVD), multiple-input multiple-output (MIMO), block diagonalization.

\section{INTRODUCTION}

In multiuser downlink multiple-input multi-output (MIMO) communications, the base-station (BS) will communicate with several users simultaneously over the same frequency band [1]. In this case, the beamformer based spatial multiplexer should be designed to suppress inter-user interference (IUI) and to maximize signal-to-noise-ratio (SNR) of each user.

There are three main classes of solutions for multiuser MIMO downlink communications. The first class of solutions uses "dirty paper coding" [2], which is a nonlinear precoding scheme for interference precancellation proposed by Costa [3]. The underlying idea is that when a transmitter has advance knowledge of the interference of a channel, an appropriate code can be designed to compensate for it, and the capacity of the channel is the same as if there was no interference. However, DPC is complicated to realize.

The second class of solutions perfectly cancels IUI for each user using linear precoding at the transmitter [4]-[8]. These schemes impose a restriction on the system configuration in terms of number of antennas. In [5], [8], block diagonalization is used to eliminate IUI, leaving each user to deal with interference among its own data stream. The zero forcing (ZF) approach in [4] is equivalent to using block diagonalization

This work has been supported by the Engineering and Physical Sciences Research Council of the UK under the grant, EPSRC E041817. to cancel IUI. However, ZF requires the number of transmit antennas at the BS to be larger than the sum of receive antennas of all users.

The third class of solutions employs linear filters, both at the transmitter and the receiver, and it generally uses iterative algorithms to optimize the weights of the precoders and decoders to maximize various criteria such as the output signalto-interference-plus-noise ratio (SINR) [9]-[13], the signal-toleakage ratio (SLR) [14]-[16], or signal-to-leakage-plus-noise ratio (SLNR) [17], [18]. The problem of transmitted power minimization with SINR constraints has been comprehensively studied in [19]-[21].

In this paper, we propose a hybrid nullspace-directed geometric mean decomposition (GMD) scheme. The reason we use GMD is that it has the potential to provide equal bit error rate (BER) performance for all subchannels [22], [23]. Considering that the iterative algorithm proposed in [7] is not guaranteed to converge, this paper proposes a mixed algorithm, where if the iterative algorithm does not converge within a specific number of iterations, the non-iterative algorithm will replace it to obtain the weight coefficients. In addition, we utilize the conventional Vertical Bell Labs. space time (VBLAST) [23], [24] scheme in the simulation.

This paper is organized as follows. A multiuser multistream MIMO system is described in the next section. After the iterative nullspace-directed GMD is proposed in Section III, the non-iterative nullspace-directed GMD and mixed nullspacedirected GMD are proposed in Section IV. Section V presents the simulation results, and the conclusion is drawn in Section VI.

\section{System Model}

Consider a downlink communication system with a BS communicating with $K$ users. There are $N_{t}$ antennas at the BS and $N_{r, i}$ receiving antennas for the $i$ th user. Let $\mathbf{s}_{i} \in \mathbb{C}^{N_{m, i} \times 1}$ denote the transmitted data symbol vector intended for user $i$, where $N_{m, i}$ denotes the number of data streams transmitted simultaneously for user $i$ such that

$$
\mathbf{s}_{i}=\left[\begin{array}{llll}
s_{i, 1} & s_{i, 2} & \cdots & s_{i, N_{m, i}}
\end{array}\right]^{T}
$$

where the superscript $T$ denotes transpose.

Before being transmitted over the channel, this symbol vector is multiplied by a precoding matrix $\mathbf{W}_{i} \in \mathbb{C}^{N_{t} \times N_{m, i}}$. Hence, the overall transmitted vector $\mathbf{x} \in \mathbb{C}^{N_{t} \times 1}$ by the 
transmit antennas can be described as

$$
\mathbf{x}=\sum_{k=1}^{K} \mathbf{W}_{k} \mathbf{s}_{k}
$$

Then, the input-output relationship of the channel can be written as

$$
\mathbf{y}_{i}=\mathbf{H}_{i} \mathbf{x}+\mathbf{n}_{i}
$$

where $\mathbf{y}_{i} \in \mathbb{C}^{N_{m, i} \times 1}$ denotes the received vector by the antennas of user $i ; \mathbf{H}_{i} \in \mathbb{C}^{N_{r, i} \times N_{t}}$ denotes the channel matrix from the transmit antennas to the receive antennas of user $i$; $\mathbf{n}_{i} \in \mathbb{C}^{N_{r, i} \times 1}$ denotes the noise vector, whose elements are zero-mean circular additive Gaussian random variables with variance $\sigma_{i}^{2}$, and $E\left\{\mathbf{n}_{i} \mathbf{n}_{i}^{H}\right\}=\sigma_{i}^{2} \mathbf{I}_{N_{m, i}}$, where $\mathbf{I}_{N_{m, i}}$ is an $N_{m, i} \times N_{m, i}$ identity matrix and the superscript $H$ denotes conjugate transpose.

The decoding function $f(\cdot)$ of user $i$ is defined as

$$
\hat{\mathbf{s}}_{i}=f\left(\mathbf{y}_{i}\right)
$$

where $\hat{\mathbf{s}}_{i} \in \mathbb{C}^{N_{m, i} \times 1}$ denotes the decoded signal.

\section{Iterative NullspaCe-DiRected GMD}

The iterative nullspace-directed SVD for a downlink multiuser multistream MIMO channel has been proposed in [7]. The multiuser signals have been projected onto orthogonal subspaces to eliminate IUI. Then the SVD has been used to decompose the multistream channel into a set of single input single output (SISO) subchannels. Therefore, each subchannel has different signal-to-noise ratios (SNR), and subchannels with the lowest SNR will limit the overall BER performance of this system. In order to obtain equal BER, we propose to replace the SVD with the GMD [22] so that identical SNR for all subchannels can be achieved. We use the VBLAST with QR decomposition (VBLAST-QR) technique to decode the received signals. In the scheme of iterative nullspace-directed GMD, the multiuser signals are projected first onto orthogonal subspaces to eliminate IUI; and GMD is used to guarantee identical SNR for all subchannels for each user. The received signals are also decoded by VBLAST-QR.

\section{A. GMD}

The GMD for MIMO has been proposed in [22] using the following optimization framework.

$$
\begin{array}{cl}
\max _{P, Q} \min & \left\{r_{i i}: 1 \leqslant i \leqslant L\right\} \\
\text { subject to } \quad \mathbf{H}^{\prime}=\mathbf{Q R P}^{H}, \mathbf{Q}^{H} \mathbf{Q}=\mathbf{I}, \mathbf{P}^{H} \mathbf{P}=\mathbf{I} \\
\mathbf{R} \in \mathbb{R}^{L \times L} \\
r_{i i}>0,1 \leqslant i \leqslant L \\
r_{i j}=0, \text { for } i>j
\end{array}
$$

where $\mathbf{H}^{\prime}$ denotes the channel matrix; $L$ denotes the rank of $\mathbf{H}^{\prime}$; the unitary matrices $\mathbf{Q}$ and $\mathbf{P}$ denote the linear operations performed at the receiver and transmitter, respectively; $\mathbf{R}$ denotes an upper triangular matrix; $r_{i j}$ denotes the $(i, j)$ th element of matrix $\mathbf{R}$. The diagonal elements of $\mathbf{R}$ are given by

$$
r_{i i}=\bar{\lambda}_{\mathbf{H}} \triangleq\left(\prod_{n=1}^{L} \lambda_{\mathbf{H}, n}\right)^{1 / L}, 1 \leqslant i \leqslant L
$$

where $\lambda_{\mathbf{H}, n}(1 \leqslant n \leqslant L)$ denote the singular values of matrix $\mathbf{H}$. Then, the diagonal elements $r_{i i}$ of matrix $\mathbf{R}$ are equal to the geometric means of the singular values of $\mathbf{H}$. Hence, GMD is asymptotically optimal in terms of the channel capacity for high SNR [23].

GMD starts with the SVD $\mathbf{H}^{\prime}=\mathbf{U} \boldsymbol{\Lambda} \mathbf{V}^{H}$, and then generates the matrix $\mathbf{Q}_{0}, \mathbf{P}_{0}$ and $\mathbf{R}$, which satisfy $\mathbf{Q}_{0}^{H} \mathbf{\Lambda} \mathbf{P}_{0}=\mathbf{R}$. Hence, matrix $\mathbf{H}^{\prime}$ is composed as $\mathbf{H}^{\prime}=\mathbf{Q R} \mathbf{P}^{H}$, where $\mathbf{Q}=\mathbf{U Q}_{0}$ and $\mathbf{P}=\mathbf{V P}_{0}[22]$.

To achieve high SNR in a multistream MIMO system, we modify the GMD as follows

1) Decompose $\mathbf{H}^{\prime}$ as $\mathbf{H}^{\prime}=\mathbf{U} \boldsymbol{\Lambda} \mathbf{V}^{H}$.

2) Let $\hat{\mathbf{U}}=\left.\mathbf{U}\right|_{1 \leftrightarrow N_{m}}$ and $\hat{\mathbf{V}}=\left.\mathbf{V}\right|_{1 \leftrightarrow N_{m}}$, where $N_{m}$ denotes the number of streams; the notation $\left.\cdot\right|_{1 \leftrightarrow N_{m}}$ denotes collecting the column vectors that correspond to the $N_{m}$ largest singular values. $\hat{\boldsymbol{\Lambda}}$ is a diagonal matrix, and its diagonal elements are the $N_{m}$ largest singular values of $\mathbf{H}^{\prime}$.

3) Then, $\mathbf{R}=\hat{\mathbf{Q}}_{0}^{T} \hat{\mathbf{\Lambda}} \hat{\mathbf{P}}_{0}, \mathbf{Q}=\hat{\mathbf{U}} \hat{\mathbf{Q}}_{0}$ and $\mathbf{P}=\hat{\mathbf{V}} \hat{\mathbf{P}}_{0}$.

\section{B. Orthogonal Space-Division Multiplexing}

Here, GMD is used to replace the SVD in the iterative nullspace-directed SVD, which was proposed in [7]. Suppose, in the receiver, the received signal $\mathbf{y}_{i}$ is premultiplied by a matrix $\mathbf{T}_{i}$, i.e.,

$$
\mathbf{y}_{i}^{\prime}=\mathbf{T}_{i} \mathbf{y}_{i}
$$

where vector $\mathbf{y}_{i}^{\prime} \in \mathbb{C}^{N_{m, i} \times 1}$ and matrix $\mathbf{T}_{i} \in \mathbb{C}^{N_{m, i} \times N_{r, i}}$.

Substituting (2) and (3) into (6), we obtain

$$
\begin{aligned}
{\left[\begin{array}{c}
\mathbf{y}_{1}^{\prime} \\
\mathbf{y}_{2}^{\prime} \\
\vdots \\
\mathbf{y}_{K}^{\prime}
\end{array}\right]=} & {\left[\begin{array}{cccc}
\mathbf{T}_{1} \mathbf{H}_{1} \mathbf{W}_{1} & \mathbf{T}_{1} \mathbf{H}_{1} \mathbf{W}_{2} & \cdots & \mathbf{T}_{1} \mathbf{H}_{1} \mathbf{W}_{K} \\
\mathbf{T}_{2} \mathbf{H}_{2} \mathbf{W}_{1} & \mathbf{T}_{2} \mathbf{H}_{2} \mathbf{W}_{2} & \cdots & \mathbf{T}_{2} \mathbf{H}_{2} \mathbf{W}_{K} \\
\vdots & \vdots & \ddots & \vdots \\
\mathbf{T}_{K} \mathbf{H}_{K} \mathbf{W}_{1} & \mathbf{T}_{K} \mathbf{H}_{K} \mathbf{W}_{2} & \cdots & \mathbf{T}_{K} \mathbf{H}_{K} \mathbf{W}_{K}
\end{array}\right] } \\
& {\left[\begin{array}{c}
\mathbf{s}_{1} \\
\mathbf{s}_{2} \\
\vdots \\
\mathbf{s}_{K}
\end{array}\right]+\left[\begin{array}{c}
\mathbf{n}_{1}^{\prime} \\
\mathbf{n}_{2}^{\prime} \\
\vdots \\
\mathbf{n}_{K}^{\prime}
\end{array}\right] }
\end{aligned}
$$

where $\mathbf{n}_{i}^{\prime}=\mathbf{T}_{i} \mathbf{n}_{i}$.

The orthogonal space-division multiplexing scheme is to eliminate IUI by making $\mathbf{T}_{i} \mathbf{H}_{i} \mathbf{W}_{j}$ equal to a zero matrix, if $i \neq j$, i.e.,

$$
\mathbf{W}_{i} \in \operatorname{null}\left\{\tilde{\mathbf{H}}_{i}^{e}\right\}
$$

where

$$
\tilde{\mathbf{H}}_{i}^{e}=\left[\begin{array}{c}
\mathbf{T}_{1} \mathbf{H}_{1} \\
\vdots \\
\mathbf{T}_{i-1} \mathbf{H}_{i-1} \\
\mathbf{T}_{i+1} \mathbf{H}_{i+1} \\
\vdots \\
\mathbf{T}_{K} \mathbf{H}_{K}
\end{array}\right] \in \mathbb{C}^{\left(\sum_{k=1, k \neq i}^{K} N_{m, k}\right) \times N_{t}}
$$


Then, $\mathbf{y}_{i}^{\prime}$ can be simplified as

$$
\mathbf{y}_{i}^{\prime}=\mathbf{T}_{i} \mathbf{H}_{i} \mathbf{W}_{i} \mathbf{s}_{i}+\mathbf{n}_{i}^{\prime}
$$

Let $\mathbf{G}_{i} \in \mathbb{C}^{N_{t} \times\left(N_{t}-\sum_{k=1, k \neq i}^{K} N_{m, k}\right)}$ denote the orthonormal basis of null $\left\{\tilde{\mathbf{H}}_{i}^{e}\right\}$. Decomposing $\mathbf{H}_{i} \mathbf{G}_{i}$ by GMD, we obtain

$$
\mathbf{H}_{i} \mathbf{G}_{i}=\mathbf{Q}_{i} \mathbf{R}_{i} \mathbf{P}_{i}^{H}
$$

where $\mathbf{Q}_{i} \in \mathbb{C}^{N_{r, i} \times N_{m, i}}, \mathbf{R}_{i} \in \mathbb{C}^{N_{m, i} \times N_{m, i}}$ and $\mathbf{P}_{i} \in$ $\mathbb{C}^{\left(N_{t}-\sum_{k=1, k \neq i}^{K} N_{m, k}\right) \times N_{m, i}}$.

Let the weight matrix

$$
\mathbf{W}_{i}=\mathbf{G}_{i} \mathbf{P}_{i}
$$

Substituting (10) and (11) into (9), yields

$$
\mathbf{y}_{i}^{\prime}=\mathbf{T}_{i} \mathbf{Q}_{i} \mathbf{R}_{i} \mathbf{s}_{i}+\mathbf{n}_{i}^{\prime}
$$

Likewise, using (6) in (12), and simplifying, we obtain

$$
\mathbf{y}_{i}=\mathbf{Q}_{i} \mathbf{R}_{i} \mathbf{s}_{i}+\mathbf{n}_{i}
$$

\section{VBLAST-QR}

We consider a VBLAST scheme with a simple suboptimal receiver interface [25]. The receiver is based on estimating a signal with the largest SNR first, and then canceling its interference component successively from the rest of the signals. This process is repeated until all of the signals are estimated. Here, we consider the decoding method of VBLAST based on QR decomposition. Considering $\mathbf{H}_{i} \mathbf{W}_{i}=\mathbf{Q}_{i} \mathbf{R}_{i}$ by (10) and (11), $\mathbf{Q}_{i}$ is an unitary matrix and $\mathbf{R}_{i}$ is an upper triangular matrix, then the QR decomposition of $\mathbf{H}_{i} \mathbf{W}_{i}$ is $\mathbf{Q}_{i}$ and $\mathbf{R}_{i}$.

Let

$$
\tilde{\mathbf{y}}_{i}=\mathbf{Q}_{i}^{H} \mathbf{y}_{i}
$$

where $\tilde{\mathbf{y}}_{i} \in \mathbb{C}^{N_{m, i} \times 1}$. Substituting (13) into (14), we obtain

$$
\tilde{\mathbf{y}}_{i}=\mathbf{R}_{i} \mathbf{s}_{i}+\tilde{\mathbf{n}}_{i}
$$

where $\tilde{\mathbf{n}}_{i}=\mathbf{Q}_{i}^{H} \mathbf{n}_{i}$, and $\tilde{\mathbf{n}}_{i} \in \mathbb{C}^{N_{m, i} \times 1}$. Since $\mathbf{Q}_{i}$ is an unitary matrix, the $\tilde{\mathbf{n}}_{i}$ has the same statistical properties as $\mathbf{n}_{i}$. Expanding (15) yields

$$
\begin{aligned}
{\left[\begin{array}{c}
\tilde{y}_{i, 1} \\
\tilde{y}_{i, 2} \\
\vdots \\
\tilde{y}_{i, N_{m, i}}
\end{array}\right]=} & {\left[\begin{array}{cccc}
r_{i, 11} & r_{i, 12} & \cdots & r_{i, 1 N_{m, i}} \\
0 & r_{i, 22} & \cdots & r_{i, 2 N_{m, i}} \\
\vdots & \vdots & \ddots & \vdots \\
0 & 0 & \cdots & r_{i, N_{m, i} N_{m, i}}
\end{array}\right] } \\
& {\left[\begin{array}{c}
s_{i, 1} \\
s_{i, 2} \\
\vdots \\
s_{i, N_{m, i}}
\end{array}\right]+\left[\begin{array}{c}
\tilde{n}_{i, 1} \\
\tilde{n}_{i, 2} \\
\vdots \\
\tilde{n}_{i, N_{m, i}}
\end{array}\right] }
\end{aligned}
$$

Ignoring the error-propagation effect, (16) can be decomposed into

$$
\tilde{y}_{i, j}=r_{i, j j} s_{i, j}+\tilde{n}_{i, j}
$$

Then the estimated $\hat{s}_{i, j}$ becomes

$$
\hat{s}_{i, j}=\frac{1}{r_{i, j j}} \tilde{y}_{i, j}=s_{i, j}+\frac{1}{r_{i, j j}} \tilde{n}_{i, j}
$$

Because $r_{i, 11}=\cdots=r_{i, N_{m, i} N_{m, i}}$ and $\tilde{n}_{i, j} \in \aleph\left(0, \sigma_{i}^{2}\right)$, $j=1, \ldots, N_{m, i}$, all the subchannels of the $i$ th user have identical SNR. The sequential signal detection is performed as follows:

$$
\begin{aligned}
& \text { for } j=N_{m, i}:-1: 1 \\
& \hat{s}_{i, j}=C\left[\left(\tilde{y}_{i, j}-\sum_{l=j+1}^{N_{m, i}} r_{i, j l} \hat{s}_{i, l}\right) / r_{i, j j}\right]
\end{aligned}
$$

end

where $C$ means mapping to the nearest symbol constellation.

\section{Iterative Nullspace-Directed GMD}

Using the above solutions, the iterative nullspace-directed GMD is formulated as follows.

1) Initialize $\mathbf{T}_{i}, \forall i$,

$$
\mathbf{T}_{i}=\left[\begin{array}{ll}
\mathbf{I}_{N_{m, i} \times N_{r, i}} & \mathbf{0}_{N_{m, i} \times\left(N_{r, i}-N_{m, i}\right)}
\end{array}\right]
$$

where $N_{m, i} \leqslant N_{r, i}$.

2) For each user, calculate the orthonormal basis of $\operatorname{null}\left\{\tilde{\mathbf{H}}_{i}^{e}\right\}, \mathbf{G}_{i}$. By the GMD of $\mathbf{H}_{i} \mathbf{G}_{i}$, we can obtain $\mathbf{Q}_{i}$, $\mathbf{R}_{i}$ and $\mathbf{P}_{i}$. And then use $\mathbf{T}_{i}=\mathbf{Q}_{i}^{H}$ to update matrix $\mathbf{T}_{i}$.

3) Calculate the error

$$
\varepsilon=\sum_{i=1}^{K}\left\|\tilde{\mathbf{H}}_{i}^{e} \mathbf{W}_{\mathbf{i}}\right\|_{F}^{2}
$$

where $\|\cdot\|_{F}$ denotes the Frobenius norm. If $\varepsilon \leqslant \varepsilon_{0}\left(=10^{-12}\right.$ typically), go to Step 4); otherwise, go back to Step 2).

4) Obtain the optimal precoding matrix $\mathbf{W}_{i}$ using (11), and then make $\left\|\mathbf{W}_{i}^{(j)}\right\|_{F}^{2}=1$, where $\mathbf{W}_{i}^{(j)}$ is the $j$ th column of $\mathbf{W}_{i}$ to satisfy the power constraint.

In the transmitter, the resulting $\mathbf{W}_{i}$ is used as the precoding matrix. In the receiver, VBLAST-QR is directly used to decode the received signal.

\section{Mixed Nullspace-Directed GMD}

Since iterative nullspace-directed GMD can not guarantee convergence, we propose a mixed nullspace-directed GMD. When the iterative algorithm does not converge within a specific number of iterations, the non-iterative algorithm will be replaced to obtain the weight matrices.

\section{A. Non-Iterative Nullspace-Directed GMD}

The difference between non-iterative nullspace-directed GMD and iterative nullspace-directed GMD is that the iterative nullspace-directed GMD firstly initializes the matrix $\mathbf{T}_{i}$ with an identity matrix, and then updates it using the result of GMD until the IUI is controlled within a specified error range; while the non-iterative nullspace-directed GMD does not consider the matrix $\mathbf{T}_{i}$ when it projects the multiuser signals onto orthogonal subspaces to eliminate IUI. Hence, the non-iterative algorithm does not need the iterative process. 
Substituting (2) into (3) and expanding, we obtain

$$
\begin{aligned}
{\left[\begin{array}{c}
\mathbf{y}_{1} \\
\mathbf{y}_{2} \\
\vdots \\
\mathbf{y}_{K}
\end{array}\right]=} & {\left[\begin{array}{cccc}
\mathbf{H}_{1} \mathbf{W}_{1} & \mathbf{H}_{1} \mathbf{W}_{2} & \cdots & \mathbf{H}_{1} \mathbf{W}_{K} \\
\mathbf{H}_{2} \mathbf{W}_{1} & \mathbf{H}_{2} \mathbf{W}_{2} & \cdots & \mathbf{H}_{2} \mathbf{W}_{K} \\
\vdots & \vdots & \ddots & \vdots \\
\mathbf{H}_{K} \mathbf{W}_{1} & \mathbf{H}_{K} \mathbf{W}_{2} & \cdots & \mathbf{H}_{K} \mathbf{W}_{K}
\end{array}\right] } \\
& {\left[\begin{array}{c}
\mathbf{s}_{1} \\
\mathbf{s}_{2} \\
\vdots \\
\mathbf{s}_{K}
\end{array}\right]+\left[\begin{array}{c}
\mathbf{n}_{1} \\
\mathbf{n}_{2} \\
\vdots \\
\mathbf{n}_{K}
\end{array}\right] }
\end{aligned}
$$

To eliminate IUI in (21), $\mathbf{H}_{i} \mathbf{W}_{j}$ should be set to a zero matrix, if $i \neq j$. It means that $\mathbf{W}_{i}$ is located in the nullspace of $\tilde{\mathbf{H}}_{i}$, where

$$
\tilde{\mathbf{H}}_{i}=\left[\begin{array}{c}
\mathbf{H}_{1} \\
\vdots \\
\mathbf{H}_{i-1} \\
\mathbf{H}_{i+1} \\
\vdots \\
\mathbf{H}_{K}
\end{array}\right] \in \mathbb{C}^{\left(\sum_{k=1, k \neq i}^{K} N_{r, k}\right) \times N_{t}}
$$

Then, (21) can be simplified as

$$
\mathbf{y}_{i}=\mathbf{H}_{i} \mathbf{W}_{i} \mathbf{s}_{i}+\mathbf{n}_{i}
$$

Let $\mathbf{G}_{i} \in \mathbb{C}^{N_{t} \times\left(N_{t}-\sum_{k=1, k \neq i}^{K} N_{r, k}\right)}$ denote the orthonormal basis of null $\left\{\tilde{\mathbf{H}}_{i}\right\}$. Decomposing $\mathbf{H}_{i} \mathbf{G}_{i}$ by GMD, we can obtain

$$
\mathbf{H}_{i} \mathbf{G}_{i}=\mathbf{Q}_{i} \mathbf{R}_{i} \mathbf{P}_{i}^{H}
$$

where $\mathbf{Q}_{i} \in \mathbb{C}^{N_{r, i} \times N_{m, i}}, \mathbf{R}_{i} \in \mathbb{C}^{N_{m, i} \times N_{m, i}}$ and $\mathbf{P}_{i} \in$ $\mathbb{C}^{\left(N_{t}-\sum_{k=1, k \neq i}^{K} N_{r, k}\right) \times N_{m, i}}$.

Since $\mathbf{W}_{i}$ is in the nullspace of $\tilde{\mathbf{H}}_{i}, \mathbf{W}_{i}$ is the combination of each column of $\mathbf{G}_{i}$. Hence, $\mathbf{W}_{i}$ can be defined as

$$
\mathbf{W}_{i}=\mathbf{G}_{i} \mathbf{P}_{i}
$$

Substituting (23) and (24) into (22) yields

$$
\mathbf{y}_{i}=\mathbf{Q}_{i} \mathbf{R}_{i} \mathbf{s}_{i}+\mathbf{n}_{i}
$$

Then we can use the VBLAST-QR mentioned in section III to decode the received signal $\mathbf{y}_{i}$.

\section{B. Mixed Nullspace-Directed GMD}

If the iterative nullspace-directed GMD does not converge within a specified number of iterations, the received signal could have significant IUI resulting in large BER. In order to avoid this problem, we propose a mixed nullspace-directed GMD. When the iterative nullspace-directed GMD does not converge within a specific number of iterations, the noniterative nullspace-directed GMD will be replaced the iterative algorithm. Furthermore, the computational complexity of the non-iterative nullspace-directed GMD is just similar to that of one iteration of the iterative nullspace-directed GMD. Then the mixed scheme will not bring extra computation.

\section{Simulation}

In this section, the multiuser multistream MIMO system is simulated to evaluate the performance of the proposed

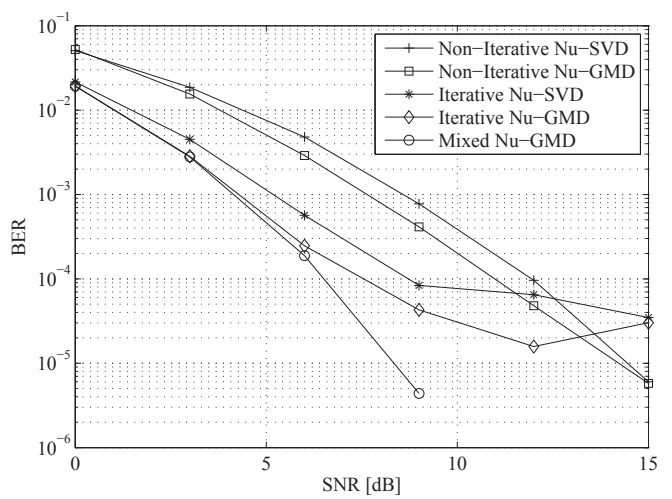

Fig. 1. A multiuser MIMO system with $N_{t}=6$ transmit antennas and $K=2$ users, each equipped with $N_{m, i}=2$ streams and $N_{r, i}=3$ receive antennas.

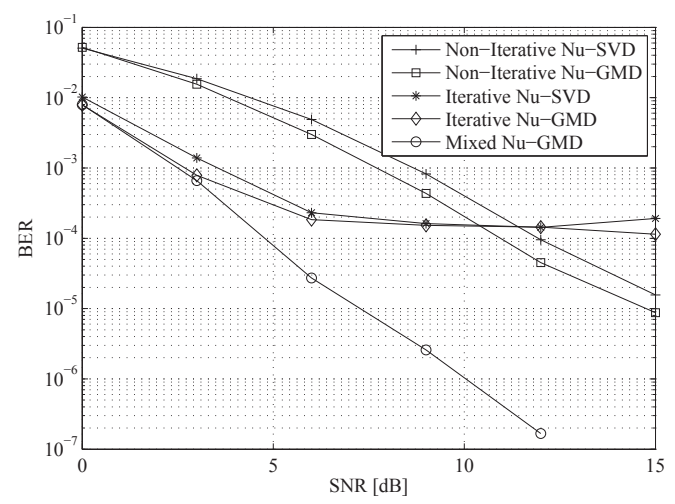

Fig. 2. A multiuser MIMO system with $N_{t}=9$ transmit antennas and $K=3$ users, each equipped with $N_{m, i}=2$ streams and $N_{r, i}=3$ receive antennas.

mixed nullspace-directed GMD scheme. A quadrature-phaseshift keying (QPSK) modulation has been used. The flat fading MIMO channel, whose elements are zero mean complex Gaussian random variables with unity variance, is fixed for 100 symbols and 10,000 independent channels are used to obtain the average BER for various SNRs. The noise variance per antenna is assumed the same for all users, $\sigma_{1}^{2}=\ldots=$ $\sigma_{K}^{2}=\sigma^{2}$.

We compare the proposed mixed nullspace-directed GMD (Mixed Nu-GMD) with four other schemes: the non-iterative nullspace-directed SVD (Non-Iterative Nu-SVD), non-iterative nullspace-directed GMD (Non-Iterative Nu-GMD) proposed in Section IV, iterative nullspace-directed SVD (Iterative NuSVD) proposed in [7] and iterative nullspace-directed GMD (Iterative Nu-GMD) proposed in Section III. All these schemes use VBLAST-QR as the decoder. The difference between non-iterative $\mathrm{Nu}-\mathrm{SVD}$ and non-iterative Nu-GMD is in the decomposition of $\mathbf{H}_{i} \mathbf{G}_{i}$ in (23). The non-iterative Nu-GMD uses GMD while non-iterative Nu-SVD uses SVD in (23).

Fig. 1 shows the BER curves for the following antenna configuration: $N_{t}=6, K=2, N_{m, i}=2, N_{r, i}=3$ and $i=1, \ldots,=K$. The antenna configuration in Fig. 2 is 
$N_{t}=9, K=3, N_{m, i}=2, N_{r, i}=3$ and $i=1, \ldots,=K$.

The two figures show that, for the same scheme, the GMD is better than SVD for reducing the BER. For example, in Fig. 1, when $\mathrm{SNR}=6 \mathrm{~dB}$, the BER values of non-iterative $\mathrm{Nu}-\mathrm{GMD}$ and iterative Nu-GMD are $2.89 \times 10^{-3}$ and $2.46 \times$ $10^{-4}$, respectively; while the BER values of corresponding schemes based on SVD are $4.80 \times 10^{-3}$ and $5.66 \times 10^{-4}$, respectively. When the SNR is small, the iterative schemes are better than the non-iterative schemes. But, with an increase in $\mathrm{SNR}$, the BER values of the iterative schemes almost remain unchanged. It is obvious in Fig. 2 that: 1)When SNR is less than $10.5 \mathrm{~dB}$, the BER values of iterative schemes are less than corresponding BER of non-iterative schemes; 2)When SNR is large than $11.4 \mathrm{~dB}$, the BER values of iterative schemes are large than corresponding BER of non-iterative schemes; 3) When SNR is large than $6 \mathrm{~dB}$, BER of iterative schemes remain between $2.3 \times 10^{-4}$ and $1 \times 10^{-4}$. It means that the BER of iterative schemes will not decrease with an increase in SNR, if SNR is larger than certain value. In addition, the iterative $\mathrm{Nu}-\mathrm{SVD}$ and iterative $\mathrm{Nu}-\mathrm{GMD}$ will not be guaranteed to converge, and the mixed Nu-GMD will automatically switch to the non-iterative $\mathrm{Nu}-\mathrm{GMD}$ algorithm if the iterative $\mathrm{Nu}-$ GMD does not converge. As shown in Fig. 1 and Fig. 2, the mixed $\mathrm{Nu}-\mathrm{GMD}$ outperforms the other four schemes.

\section{CONCLUSION}

In this paper, we proposed GMD-based spatial multiplexing for a multiuser multistream MIMO system in combination with a block diagonalization method, to yield two precoding schemes for downlink beamforming. One is the iterative nullspace-directed GMD and the other is the non-iterative nullspace-directed GMD. Considering their advantages and disadvantages, the mixed nullspace-directed GMD has also been proposed. In addition, we utilized the conventional VBLAST as the decoder. The simulation results show that the mixed nullspace-directed GMD outperforms the iterative nullspace-directed GMD and non-iterative nullspace-directed GMD. Moreover, the performances of the iterative nullspacedirected GMD and the non-iterative nullspace-directed GMD are better than the iterative nullspace-directed SVD and noniterative nullspace-directed SVD, respectively. Furthermore, the mixed scheme will not bring extra computation since the complexity of the non-iterative nullspace-directed GMD is just similar to that of one iteration of the iterative nullspacedirected GMD.

\section{REFERENCES}

[1] Q. H. Spencer, C. B. Peel, A. L. Swindlehurst and M. Haardt, "An introduction to the multi-user mimo downlink," IEEE Communications Magazine, vol. 42, no. 10, pp. 60-67, October 2004.

[2] G. J. Foschini and A. H. Diaz, "Dirty paper coding: perturbing off the infinite dimensional lattice limit," in Proc. DIMACS Workshop on Signal Processing for Wireless Transmission, Rutgers University, Piscataway, NJ, USA, October 2002, pp. 141-160.

[3] M. Costa, "Writing on dirty paper," IEEE Transactions on Information Theory, vol. 29, no. 3, pp. 439-441, May 1983.

[4] Q. H. Spencer, A. L. Swindlehurst and M. Haardt, "Zero-forcing methods for downlink spatial multiplexing in multiuser mimo channels," IEEE Transactions on Signal Processing, vol. 52, no. 2, pp. 461-471, Feb. 2004.
[5] K.-K. Wong, R. D. Murch and K. B. Letaief, "A joint-channel diagonalization for multiuser mimo antenna systems," IEEE Transactions on Wireless Communications, vol. 2, no. 4, pp. 773-786, July 2003.

[6] L. Choi and R. D. Murch, "A transmit preprocessing technique for multiuser mimo systems using a decomposition approach," IEEE Transactions on Wireless Communications, vol. 3, no. 1, pp. 20-24, January 2004.

[7] Z. Pan, K.-K. Wong and T.-S. Ng, "Generalized multiuser orthogonal space-division multiplexing," IEEE Transactions on Wireless Communications, vol. 3, no. 6, pp. 1969-1973, November 2004.

[8] R. Chen, J. G. Andrews and R. W. Heath Jr., "Multiuser space-time block coded mimo with downlink precoding," in Proc. IEEE International Conference on Communications, Paris, France, May 2004, pp. 26892693.

[9] M. Schubert and H. Boche, "Solution of the multiuser downlink beamforming problem with individual sinr constraints," IEEE Transactions on Vehicular Technology, vol. 53, no. 1, pp. 18-28, January 2004.

[10] M. Schubert and H. Boche, "Iterative multiuser uplink and downlink beamforming under sinr constraints," IEEE Transactions on Signal Processing, vol. 53, 2005.

[11] H. Boche and M. Schubert, "A general duality theory for uplink and downlink beamforming," in Proc. IEEE 56th Vehicular Technology Conference, Vancouver, Canada, September 2002, pp. 87-91.

[12] A. M. Khachan, A. J. Tenenbaum and R. S. Adve, "Linear processing for the downlink in multiuser mimo systems with multiple data streams," in Proc. IEEE International Conference on Communications, Rutgers University, Piscataway, NJ, USA, June 2006, pp. 4113-4118.

[13] P. Ma and W. Wang, "A multiuser beamforming scheme for downlink spatial multiplexing mimo," in Proc. 18th Annual IEEE International Symposium on Personal, Indoor and Mobile Radio Communications, Athens, Greece, September 2007, pp. 1-5.

[14] A. Tarighat, M. Sadek and A. H. Sayed, "A multi user beamforming scheme for downlink mimo channels based on maximizing signal-toleakage ratios," in Proc. IEEE International Conference on Acoustics, Speech, and Signal Processing, Philadelphia, USA, March 2005, pp. 1129-1132.

[15] M. C. H. Lim, M. Ghogho and D. C. McLernon, "Spatial multiplexing in the multi-user mimo downlink based on signal-to-leakage ratios," in Proc. IEEE Global Telecommunications Conference, Washington, DC, USA, November 2007, pp. 3634-3638.

[16] V. Sharma and S. Lambotharany, "Multiuser downlink mimo beamforming using an iterative optimization approach," in Proc. IEEE 64th Vehicular Technology Conference, Montreal, Canada, September 2006, pp. $1-5$.

[17] M. Sadek, A. Tarighat and A. H. Sayed, "Active antenna selection in multiuser mimo communications," IEEE Transactions on Signal Processing, vol. 55, no. 4, pp. 1498-1510, April 2007.

[18] M. Sadek, A. Tarighat and A. H. Sayed, "A leakage-based precoding scheme for downlink multi-user mimo channels," IEEE Transactions on Wireless Communications, vol. 6, no. 5, pp. 1711-1721, May 2007.

[19] M. Codreanu, A. Tolli, M. Juntti and M. Latva-aho, "Joint design of tx-rx beamformers in mimo downlink channel," IEEE Transactions on Signal Processing, vol. 55, no. 9, pp. 4639-4655, September 2007.

[20] S. Bongyong, R. L. Cruz and B. D. Rao, "A simple joint beamforming and power control algorithm for multi-user mimo wireless networks," in Proc. IEEE 60th Vehicular Technology Conference, Los Angeles, CA, USA, September 2004, pp. 247-251.

[21] A. Wiesel,Y. C. Eldar and S. Shamai, "Linear mimo precoders for fixed receivers," in in Proc. IEEE International Conference on Acoustics, Speech, and Signal Processing, Montreal, Canada, 17-21 May 2004, pp. 481-484.

[22] Y. Jiang, W. Hager and J. Li, "The geometric mean decomposition," Linear Algebra Its Applications, vol. 396, pp. 373-384, Feb. 2005.

[23] Y. Jiang, J. Li and W. W. Hager, "Joint transceiver design for mimo communications using geometric mean decomposition," IEEE Transactions on Signal Processing, vol. 53, no. 10, pp. 3791-3803, October 2005.

[24] G. J. Foschini, G. D. Golden, R. A. Valenzuela and P. W. Wolniansky, "Simplified processing for high spectral efficiency wireless communication employing multiple-element arrays," IEEE Journal on Selected Areas in Communications, vol. 17, no. 11, pp. 1841-1852, November 1999.

[25] G. J. Foschini and M. J. Gans, "On limits of wireless communications in a fading environment when using multiple antennas," Wireless Personal Communications, vol. 6, pp. 311-335, March 1998. 